\title{
Indium Phosphide Photonic Circuits on Silicon Electronics
}

\author{
K.A. Williams, X. Liu, M. Matters-Kammerer, A. Meighan, M. Spiegelberg, \\ J.J.G.M. van der Tol, M. Trajkovic, M.J. Wale, W. Yao, X. Zhang \\ Institute of Photonic Integration and the Integrated Circuits Research Group, \\ Eindhoven University of Technology, P.O.Box 513, 5600 MB Eindhoven, \\ The Netherlands Author e-mail address: k.a.williams@tue.nl
}

\begin{abstract}
The intimate integration of photonics and electronics in transceivers facilitates energyefficiency, bandwidth acceleration and a route to radical miniaturization. We present and implement a wafer-to-wafer integration method which combines electronic and photonic foundry technologies.
\end{abstract}

\section{Introduction}

The motivation to integrate electronic and photonic systems [1] in an aligned manufacturing process is long-standing, but this now becomes a necessity with the sub-systems envisaged for future data center technologies [2], light based detection and ranging [3], and future, high-density neuromorphic and programmable photonics. High-speed, highdensity, low-energy, electrical interconnects within transceivers also enable performance advances through feedforward equalization [4], optical serialization [5], equalization [6], and driver voltage reduction [7].

Photonic transceivers require precision DC biasing and wideband impedance matching to ensure data integrity. Historically separately-designed photonic and electronic chipsets have been implemented with bias tees and $50 \Omega$ interfaces to facilitate interoperability and tractable design, but this is no longer attractive as speeds increase within a restricted energy envelope. Components need to be placed closer together within the package, towards a combined 3D assembly and ultimately within the same processed wafer. Researchers are actively exploring a range of schemes for the integration and embedding of photonic circuits within electronic systems. Silicon modulators

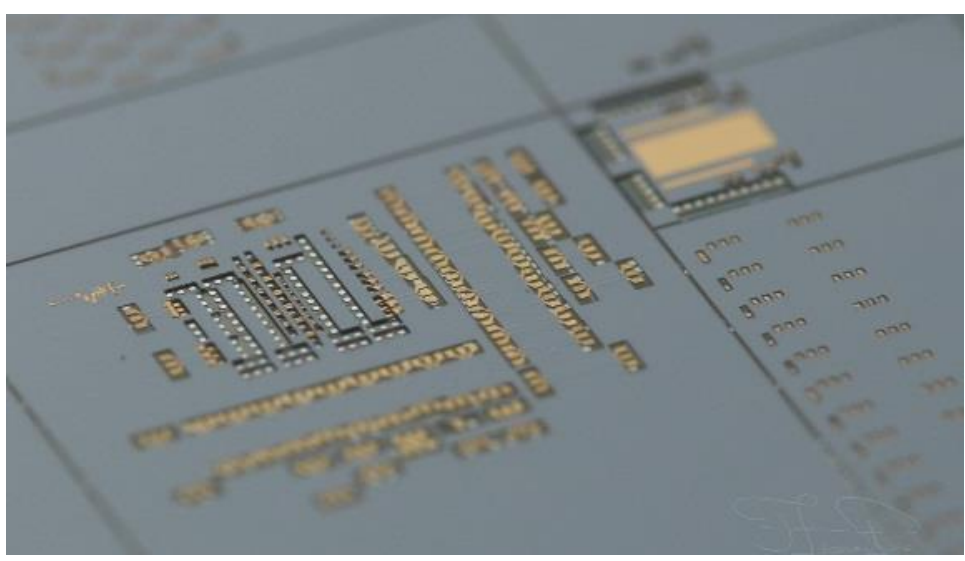

Fig 1. Indium phosphide photonic integrated circuit membrane wafer bonded to a BiCMOS wafer including the electronic interface have been integrated within silicon electronics [8-11]. Indium phosphide (InP) electronics and InP photonics have been monolithically integrated [12]. InP remains the material system of choice for efficient light sources and the highest-performance modulators in telecommunications, motivating the integration of InP circuits with silicon through a range of wafer-bonding methods [13-14]. However there has been relatively little work on the intimate integration of InP photonic integrated circuits with silicon electronics. The decision on which components to place in InP technology and silicon technology is fraught with technological and commercial uncertainties. Advanced CMOS processes evolve at such a pace and are used in such high production that it may be impractical to monolithically integrate photonic designs and re-port to the latest technology nodes. The need to produce in fabs which are not sized for the addressed markets may also add unacceptable cost. In our approach, we integrate the complete photonic integrated circuit within foundry-sourced InP and combine with electronic circuits in silicon.

The foundry model for producing integrated circuits has long existed for electronics and this is now becoming established for photonic integrated circuits [15]. It is now technologically feasible to produce application specific designs with small series foundry processes for both technologies, motivating co-designed, foundry-produced electronic and photonic systems. We use InP PICs to allow for sustained reductions in energy and footprint and increase in bandwidth as the technology platforms evolve [16], and silicon electronics as a widely accepted commercial solution with highly-developed process design kits. Our approach extends the wafer bonding methods developed for our nanophotonics platform [13] to foundry-sourced InP PIC wafers, electronics and the associated codesigned devices. We present the co-designed EAM/drivers and MZM/drivers, demonstrate interoperability, and 
present wafer-to-wafer assembly methods for increased bandwidth, with reduced loss interconnection. Figure 1 shows our first wafer-to-wafer assembly demonstration.

\section{Die-to-die assembly of co-designed circuits}

Electro-absorption modulators (EAM) have been produced using advanced foundry platforms and are demonstrated with data rates of 64Gbit/s and beyond [17-18]. These are used in combination with the bespoke drivers. A $2 \times 56$ $\mathrm{Gb} / \mathrm{s}$ PAM-4 dual-channel EAM driver has been designed and produced in a $0.25-\mu \mathrm{m}$ SiGe:C BiCMOS process [19] and delivers a maximum of $2 \mathrm{~V}$ peak-to-peak amplitude when single-ended connection is made. The driver is DCcoupled to the optical modulator, enabling a simple bond-wire connection between electronic and photonic chips. Concepts have been devised to remove the bias $\mathrm{T}$ and obtain a tunable output voltage at the output of the driver [20]. The EAM operates in the L-band at $1590 \mathrm{~nm}$, with a DC bias set at $-1.6 \mathrm{~V}$ for on-off keying non-return to zero modulation. Fig 2 shows the experimental layout and the measured eye-diagrams for a data rate of $20 \mathrm{Gbit} / \mathrm{s}$ and 36Gbit/s [21]. The reduced bandwidth relative to the individual component performance is primarily attributed to the bond-wire and bond-pad reactances.

a

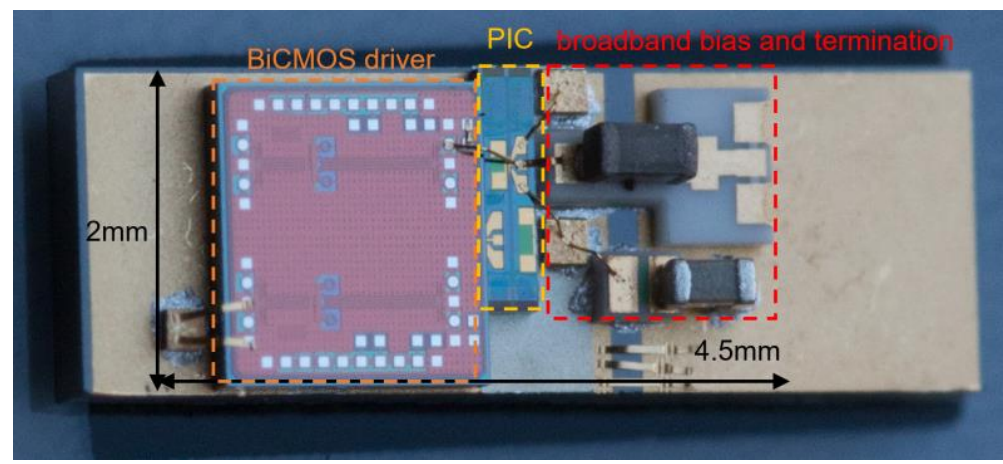

$\mathrm{b}$

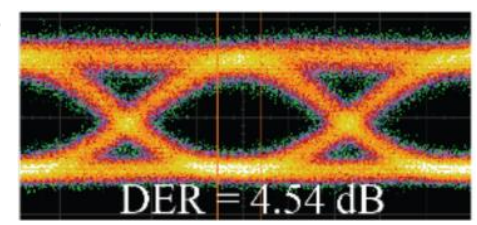

c

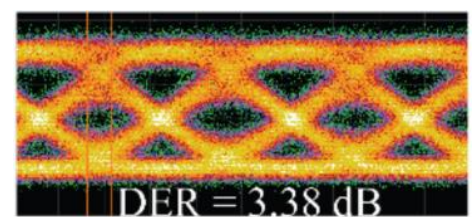

Fig 2: Direct die-to-die connection for electro-absorption modulator-driver combination operating with 20Gbit/s and $36 \mathrm{Gbit} / \mathrm{s}$ modulation.

Phase modulators using InP, multi-quantum-well (MQW) designs have been demonstrated with 40 Gbit/s MachZehnder modulator (MZM) performance with a foundry process, and have been reported elsewhere [22]. A low-power, high-efficiency, linear driver has been developed in the same $0.25 \mu \mathrm{m} \mathrm{SiGe:} \mathrm{C} \mathrm{BiCMOS} \mathrm{technology} \mathrm{for} \mathrm{this} \mathrm{class} \mathrm{of}$ optical modulator. The driver features a small-signal gain of $18 \mathrm{~dB}$, and a 3-dB bandwidth of $40 \mathrm{GHz}$ and delivers a maximum output amplitude of $4 \mathrm{~V}$ peak-to-peak to a $100 \Omega$ differential load. With inductor-peaking, this driver achieves $34 \mathrm{ps} \pm 3 \mathrm{ps}$ group delay from DC to $50 \mathrm{GHz}$. In time-domain measurements, this driver achieves a measurement platform limited symbol rate of $56 \mathrm{~Gb} / \mathrm{s} \mathrm{NRZ} \mathrm{and} 28$ Gbaud PAM4 [23]. Die-to-die interfacing will be reported.

\section{Wafer-to-wafer bonding of integrated circuits}

The wafer-to-wafer bonding of pre-defined circuits requires a methodology to (i) accommodate topography on both the electronic and photonic wafers, (ii) ensure accurate alignment between electronic and photonic circuits and (iii) provide electronic connection between circuit elements. Benzocyclobutene (BCB) polymer is used for the bonding material owing to its adhesion quality, low dielectric constant and compatibility with $\mathrm{Si}$ and InP industries [24]. A low resistance metal interconnection technique has been devised and validated for carrying the high speed RF signals [25]. Fig. 3 shows process module validation for creating electrical vias between the Si and InP. The BCB layer is opened, patterned and subsequently plated to create low resistance interconnects between the top and bottom surfaces. Details in Fig 3 show break-free connection and uniform metal thickness. Daisy-chained DC test structures show unmeasurably low excess resistance due to the vias indicating that the resistance is dominated by the gold tracks. The process module has subsequently been integrated into the full process flow to include the assembly of foundry-sourced PIC and BiCMOS wafers.

Fraunhofer HHI and NXP Semiconductor produced the integrated circuit wafers containing the ICs for the photonics and electronics respectively. The electronic ICs are as specified in section 2 . The completed wafer-scale assembly is shown in Fig 1. High-frequency test structures are on the left and a functional circuit on the right. At this specifc location an optical receiver is shown. The RF test structures give measurement limited bandwidths of $67 \mathrm{GHz}$ for lengths up to $1 \mathrm{~mm}$. 


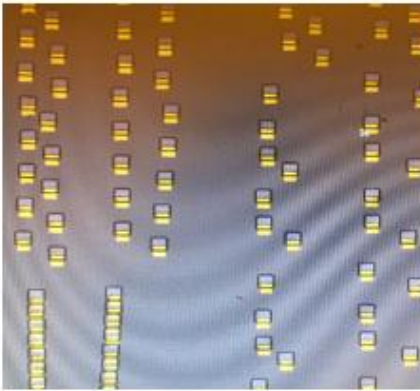

b

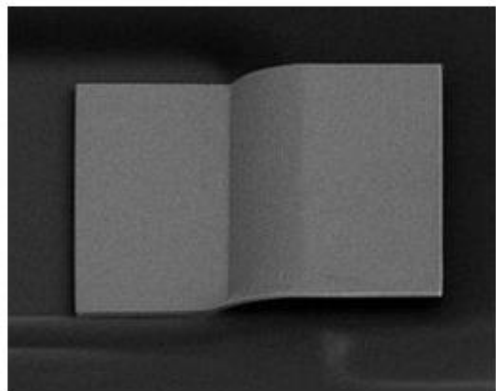

c

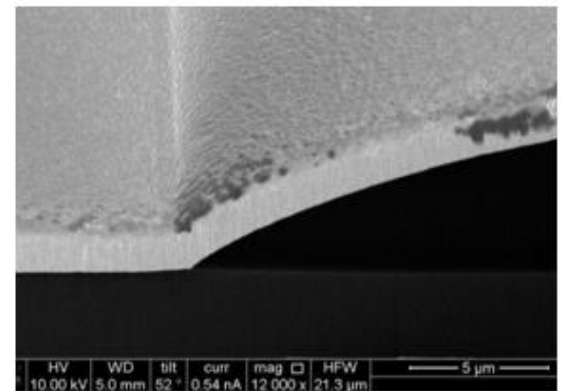

Fig 3: Through polymer vias with a) completed process module showing top-to-bottom-surface electrodes connection b) one via with dimensions $116 \times 116 \mu^{2}$ and c) detail of via to show break-free connection.

\section{Summary}

Electronic and photonic components and circuits have been designed for high speed interoperability, with leadingedge-performance using foundry processes. These have been separately assessed with 40Gbit/s performance, as well as in combination using direct die-to-die assembly with only short bond-wires and bond-pads between the circuits. This does compromise bandwidth, motivating a wafer-to-wafer method to assemble these circuits. This has been validated, and characterization of the interconnects indicates that low-loss broadband interconnection is feasible. The first wafer-to-wafer bonded, co-designed circuits have been created and insights will be presented.

\section{Summary}

The Dutch NWO Photronics, the EC Horizon 2020 WIPE and Dutch NWO Zwaartkracht projects are acknowledged.

\section{References}

[1] M.K. Smit, "Integration of photonics and electronics", IEEE International solid-state circuits conference, keynote, 2019

[2] IEEE P802.3bs 400 Gb/s Ethernet Task Force. [Online]. Available: http://www.ieee802.org/3/bs/. [Accessed: 13-Dec-2019].

[3] T. Kim et al., "A single-chip optical phased array in a wafer-scale silicon photonics / CMOS 3D-integration platform", IEEE Journal of Solid-State Circuits, 54, 11, 3061, 2019

[4] H. Li et al., "A $112 \mathrm{~Gb} / \mathrm{s}$ PAM4 transmitter with silicon photonics microring modulator and CMOS driver", OFC 2019

[5] J. Lambrecht et al., "Low-Power (1.5 pJ/b) Silicon Integrated 106 Gb/s PAM-4 Optical Transmitter", IEEE JLT, early access, 2019

[6] B. G. Lee et al., "Driver-integrated 56-Gb/s segmented electrode silicon Mach Zehnder modulator using optical-domain equalization", OFC, 2017

[7] B. G. Lee et al., "FEC-free 60-Gb/s silicon photonic link using SiGe-driver ICs hybrid-integrated with photonics-enabled CMOS", OFC 2018

[8] D. Petousi et al., "Monolithically integrated high-extinction-ratio MZM with a segmented driver in photonic BiCMOS", IEEE Photonics Technology Letters, 28, 24, 2866-2869, 2016

[9] Y. Ogiso et al., "Ultra-high bandwidth InP IQ modulator co-assembled with driver IC for beyond 100-GBd CDM",OFC 2018

[10] Z. Yong et al., "A 44Gbps high extinction ratio silicon Mach-Zehnder modulator with a 3D-integrated 28nm FD-SOI CMOS driver", OFC 2017

[11] Y. Ma et al., "An all-silicon transmitter with co-designed modulator and DC-coupled driver", OFC 2019

[12] J.K. Park et al., "Monolithic integration of InGaAsP MZI modulator and InGaAs driver MOSFET using III-V CMOS photonics", OFC 2017

[13] J.J.G.M. van der Tol et al., "Indium phosphide integrated photonics in membranes", IEEE JSTQE, 24, 1, 9, 6100809, 2018

[14] Semiconductors and Semimetals, Silicon Photonics, editors S. Lourdudoss, R.T. Chen and C. Jagadish, 99, 189-219, Elsevier, 2018

[15] The EC InPulse project enables an open access manufacturing pilot line capability for InP PICs: inpulse.jeppix.eu

[16] M.K. Smit et al., "Past, present and future of InP-based photonic integration", APL Photonics, 4, 5, 050901, 2019

[17] M. Trajkovic et al., "64Gb/s electro absorption modulator operation in InP-based active-passive generic integration platform", ECOC 2018

[18] M. Trajkovic et al., "112Gb/s PAM-4 transmission over 1.5km with an EAM in generic integration platform", OECC 2019

[19] X. Zhang et al., "Dual-channel 56 Gb/s PAM-4 electro-absorption modulator driver for 3D wafer scale packaging", 30th Asia-Pacific Microwave Conference, APMC, 237-239, Kyoto, 2018

[20] X. Zhang, X. Liu , M. Spiegelberg, A.R. van Dommele, M.K. Matters-Kammerer, "A DC-51.5 GHz electro-absorption modulator driver with tunable differential DC coupling for 3D wafer scale packaging", proceedings BCICTS, Nashville, 2019.

[21] M. Trajkovic, et al. "36 Gb/s operation of a BiCMOS driver and InP EAM using foundry platforms", ECOC 2019.

[22] W. Yao et al., "Monolithic $300 \mathrm{~Gb} / \mathrm{s}$ parallel transmitter in InP based generic photonic integration technology", JSTQE, 24, 1, 6100711, 2018

[23] X. Liu et al., "A DC to $40 \mathrm{GHz} 4-\mathrm{Vpp}$ output high-efficiency linear driver for optical communication", Asia-Pacific Microwave Conference, 1519-1521, Kyoto, 2018

[24] M. Spiegelberg et al., "BCB bonding of high topology 3 inch InP and BiCMOS wafers for integrated optical transceivers", IEEE Photonics Benelux Chapter Annual Symposium, 160-163, Brussels, 2018

[25] A. Meighan et al, "Low resistance metal interconnection for direct wafer bonding of electronic to photonic ICs", 22nd Annual Symposium of the IEEE Photonics Society Benelux Chapter, Delft, 2017 